\title{
Orientações aos fisioterapeutas para a realização de entrevistas e comunicações à imprensa durante a pandemia de COVID-19*
}

\author{
Guidelines for physiotherapists to performing interviews and communications to the press \\ during the COVID-19 pandemic*
}

\author{
VELLOSO, Marcelo; ; MATTE, Darlan Laurício²; ANDRADE, Flávio Maciel Dias de \\ COSTA, Marcelo Dourado4; MARTINEZ, Bruno Prata ${ }^{5}$; em nome Comitê COVID-19 da \\ ASSOBRAFIR.
}

\begin{abstract}
Resumo
Em tempos de pandemia a comunicação dos profissionais da saúde com a imprensa e com a população em geral é de fundamental importância. O fisioterapeuta é um dos profissionais envolvidos diretamente com os cuidados aos pacientes com COVID-19, sendo assim, tem a obrigação de esclarecer a população sobre as questões relativas ao fazer do profissional. Recomenda-se que a comunicação seja realizada com cautela, de forma respeitosa e clara, mantendo a postura profissional e sobretudo fornecendo informações embasadas em evidências cientificas, para que assim, não haja equívocos na informação.
\end{abstract}

Palavras-chave: Fisioterapia; Comunicação; COVID-19.

* Revisado por membros do Comitê COVID-19 da ASSOBRAFIR, nomeado por meio do memorando N 003/2020. Esta publicação é uma atualização parcial da Comunicação Oficial "O fisioterapeuta e sua relação com o novo betacoronavirus 2019 (2019-nCoV)", chancelada pelo Comitê COVID-19 da ASSOBRAFIR, originalmente escrita pelos mesmos autores e divulgada em 31/01/2020 no endereço eletrônico https://assobrafir.com.br/wp-content/uploads/2020/01/ASSOBRAFIR BETACORONAVIRUS-2019 v.4.pdf

1 Programa de Pós-Graduação em Ciências da Reabilitação, Universidade Federal de Minas Gerais (UFMG), Belo Horizonte, Minas Gerais, Brasil. Departamento de Fisioterapia, Universidade Federal de Minas Gerais (UFMG), Belo Horizonte, Minas Gerais, Brasil. Email: marcello.vel@gmail.com. MV - https://orcid.org/0000-0002-2352-8954

2 Programa de Pós-Graduação em Fisioterapia, Universidade do Estado de Santa Catarina (UDESC), Florianópolis, Santa Catarina, Brasil. Programa de Pós-Graduação em Ciências do Movimento Humano, Universidade do Estado de Santa Catarina (UDESC), Florianópolis, Santa Catarina, Brasil. Departamento de Fisioterapia. Universidade do Estado de Santa Catarina (UDESC), Florianópolis, Santa Catarina, Brasil. DLM - https://orcid.org/0000-0003-4650-3714

3 Universidade Católica de Pernambuco (UNICAP), Recife, Pernambuco, Brasil. FMD - https://orcid.org/0000-0001-9571-6551

${ }^{4}$ Conselho Regional de Fisioterapia e Terapia Ocupacional da 7a. Região (CREFITO-7), Salvador, Bahia, Brasil. MDC https://orcid.org/0000-0003-2164-6719

5 Programa de Pós-Graduação em Medicina e Saúde, Universidade Federal da Bahia (UFBA), Salvador, Bahia, Brasil. Colegiado de Fisioterapia, Universidade do Estado da Bahia (UNEB), Salvador, Bahia, Brasil. BPM - https://orcid.org/0000-0002-4673$\underline{8698}$ 


\section{Abstract}

In pandemic times, it is very important that health professionals communicate with the media and the general population. The physiotherapist is one of the health professionals directly involved with COVID-19 patients care. Therefore, it is necessary that physiotherapists clarify to the general population about issues related to the professional's practice. The present guidelines recommend that communication should be carried out with caution, in a respectful and clear manner, keeping a professional behaviour and providing information based on scientific evidence to avoid any misundstanding.

Keywords: Physiotherapy; Communication; COVID-19.

\section{Objetivo}

O objetivo do presente posicionamento é oferecer subsídios e orientar os fisioterapeutas na realização de entrevistas ou divulgação de materiais, incrementando a qualidade da participação do profissional nas ações junto a imprensa durante a pandemia de COVID-19. Tendo em vista a atuação do Fisioterapeuta Respiratório, Cardiovascular e de Terapia Intensiva na linha de frente do enfrentamento à doença, este precisa estar preparado para aproveitar as oportunidades de divulgação da profissão à imprensa nacional e internacional.

\section{Papel do Fisioterapeuta Respiratório, Cardiovascular e de Terapia Intensiva na COVID-19}

O fisioterapeuta, em suas diversas áreas de atuação, é um profissional indispensável no tratamento de pacientes com condições agudas e crônicas de saúde. Especialmente neste momento de enfrentamento da pandemia de COVID-19, as áreas de Fisioterapia Respiratória, Cardiovascular e de Terapia Intensiva ${ }^{1,2,3}$ estão em destaque, pois são as especialidades que se encontram na linha de frente do combate à COVID-19.

A Fisioterapia tem como objetivo preservar e restaurar a funcionalidade do paciente, seja ela orgânica ou motora, sempre assistindo o indivíduo em sua totalidade. Desta forma, as áreas de Fisioterapia Respiratória, Cardiovascular e Terapia Intensiva são complementares, embora tenham suas particularidades.

$\mathrm{Na}$ situação atual, os pacientes com COVID-19 que são encaminhados para os hospitais com quadros graves de insuficiência respiratória necessitam do atendimento do fisioterapeuta, tendo em vista que boa parte deles necessitará de internação em Unidades de Terapia Intensiva (UTI) ${ }^{4}$. Segundo a RESOLUÇÃO-RDC No 7, de 24 de FEVEREIRO DE $2010^{5}$ que dispõe sobre os requisitos mínimos para funcionamento de UTI no Brasil, os fisioterapeutas juntamente como os médicos e enfermeiros fazem parte da equipe mínima de atendimento ao paciente crítico $^{5}$.

O papel do fisioterapeuta dentro dos hospitais e das UTI compreende desde o auxílio ao paciente em mobilizar e eliminar secreções pulmonares; promoção de reexpansão pulmonar; monitorização respiratória e cardiovascular; oferta e monitoramento do suporte de oxigênio; condução e ajuste dos parâmetros da ventilação mecânica invasiva e não-invasiva; avaliação neuro-músculo-esquelética e funcional; e instituição de protocolos sistematizados de mobilização e exercícios terapêuticos precoces. Outro ponto importante consiste em favorecer o retorno dos pacientes às suas atividades funcionais o mais rapidamente possível, sendo esta abordagem iniciada ainda na UTI, continuando nas unidades 
de internação (apartamentos ou enfermarias), devendo-se sempre considerar a sua continuidade em ambulatórios e clínicas, visto que muitos pacientes apresentam a Síndrome Pós-Terapia Intensiva (também chamada de Síndrome Pós Doença Crítica), a qual pode perdurar por longo período após a alta hospitalar.

Especificamente na situação dos pacientes com COVID-19, o fisioterapeuta deve avaliar as necessidades individuais para elaborar sua conduta. Uma atuação possível é auxiliando os pacientes com tosse produtiva, com secreções abundantes de vias aéreas e que não conseguem eliminá-la de maneira independente e eficiente, por meio de técnicas de remoção de secreção, embora este sintoma seja menos comum na COVID-19, ele ocorre em $34 \%$ dos pacientes ${ }^{4}$. Pacientes de alto risco para o COVID-19 também se beneficiam da atuação do fisioterapeuta, pois aqueles que possuem comorbidades tais como doenças neuromusculares, doenças respiratórias crônicas, entre outras, frequentemente apresentam hipersecreção ou tosse ineficaz .

$\mathrm{Na}$ UTI, o fisioterapeuta também pode atuar na manutenção da permeabilidade das vias aéreas dos pacientes ventilando espontaneamente ou mecanicamente, e utilizar o posicionamento terapêutico como estratégia para expansão pulmonar, melhora do clearance mucociliar e da eficiência da tosse $e^{4}$. Os fisioterapeutas também têm papel fundamental na monitorização e ajustes do ventilador mecânica, buscando sempre a correção da troca gasosa, empregando ventilação pulmonar protetora, para preservar a função pulmonar e muscular respiratória, corroborando para redução do tempo de permanência no ventilador ${ }^{4}$.

Devido aos fatores inerentes à doença de base ou internamento, bem como à necessidade do uso de sedação e de agentes bloqueadores neuromusculares, pacientes com COVID-19 admitidos na UTI apresentam alto risco para desenvolver problemas vasculares periféricos e a polineuromiopatia do paciente crítico ou fraqueza muscular adquirida na UTI, fatores complicadores do quadro, que podem piorar a morbimortalidade ${ }^{4}$. Nessa situação, o fisioterapeuta é essencial, pois pode se antecipar aos efeitos deletérios dessas complicações, iniciando precocemente a realização de protocolos de manutenção ou recuperação do desempenho muscular e nível de funcionalidade, para minimizar sua gravidade e promover recuperação funcional mais breve.

Uma publicação recente já aponta a reabilitação como uma forma de tratamento que deverá ser oferecido aos sobreviventes para combater as consequências do COVID- $19^{6}$. Assim como os pacientes que passaram por doenças críticas, os sobreviventes da COVID-19 deverão se beneficiar da reabilitação para obter melhores resultados de saúde e funcionalidade, facilitação da alta precoce e redução do risco de reinternação ${ }^{6}$. Todos os procedimentos com a finalidade de permitir o retorno do indivíduo o mais rápido possível às atividades comuns do dia a dia e àquela relacionadas ao trabalho.

Em relação à prevenção ou minimização dos sintomas respiratórios em pacientes com suspeita ou formas leves da COVID-19, respectivamente, não existem evidências que exercícios respiratórios possam prevenir a doença ou minimizar esses sintomas. Entretanto, a realização de exercícios físicos deve ser incentivada, buscando a manutenção de um bom estado geral de saúde.

\section{Orientações para a realização de entrevistas com a imprensa}

A prerrogativa básica para participação em uma entrevista refere-se ao domínio que o profissional deve ter sobre o assunto a que se propõe falar ou argumentar, já que o conhecimento, mesmo dentro de uma única profissão, é bastante extenso e nenhum profissional é capaz de falar sobre todos os temas. 
As orientações para participação em entrevistas com a imprensa foram agrupadas em três momentos: pré-entrevista; durante a entrevista e pós-entrevista, e são apresentadas a seguir:

\section{Pré-entrevista}

1.1. Ao receber uma solicitação da imprensa, é necessária certa agilidade, devido aos prazos determinados.

1.2. Durante o diálogo com o jornalista, seja objetivo, sem utilização de termos técnicos, ou quando usá-lo, explique-os, para que haja entendimento comum. Caso exista abertura por parte do entrevistador sugira ou combine as perguntas e os questionamentos com o jornalista ou entrevistador antes da reportagem, principalmente se a mesma for ao vivo.

1.3. Seja sempre educado, mesmo em perguntas desagradáveis ou indiscretas, pois isso faz parte do trabalho dos jornalistas e nós devemos responder de forma técnica e científica, conforme nosso conhecimento atual.

1.4. Para respaldar suas descrições, use documentos, evidências; resoluções ou pareceres técnico-científicos, de modo que a informação não seja apenas fruto da sua opinião, ou das suas reflexões. Caso não tenha a informação sobre algo, se comprometa a buscar a fonte ou a resposta, conforme o conhecimento atual.

1.5. A entrevista deve ter conteúdo informativo e não formativo. Portanto, não se deve ensinar condutas ou técnicas próprias da Fisioterapia, uma vez que o público que atinge não é exclusivamente formado por profissional fisioterapeuta ou estudantes de Fisioterapia, sob pena de infração ao Código de Ética (Art. 30, inciso XII, da Resolução COFFITO No $424 / 2013)^{7}$.

1.6. Nunca passe informações sobre o estado de saúde de pacientes, sob pena de infração

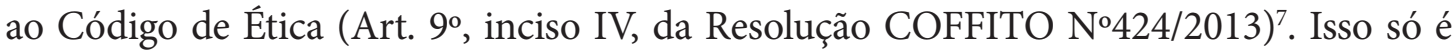
possível nas seguintes situações: demandas judiciais, inquéritos policiais, auditoria ou para a família do paciente.

1.7. Seja sincero e sempre fale a verdade, com base no conhecimento atual e nos casos em que queira emitir sua opinião. Nesta última, tenha muito cuidado, pois as repercussões negativas podem ser significativas. Atentar para o zelo pela dignidade da profissão, conforme estabelece o Art. 9º inciso II, da Resolução COFFITO No 424/20137.

1.8. De forma alguma, deve-se valer do interesse mercadológico para defender condutas terapêuticas sem a devida comprovação científica ou consideradas infalíveis.

\section{Durante a entrevista}

2.1. Respeitar as regras quando houver a necessidade de distanciamento social ou uso de equipamentos de proteção individual, devido ao risco de contaminações por contato, aerossóis ou gotículas.

2.2. Preste atenção em sua postura corporal, pois isso é de extrema importância. Evite movimentos amplos de braços, mãos e cabeça, bem como permaneça relativamente relaxado. Se a entrevista for em pé, mantenha a base corporal fixa com os pés levemente afastados; quando a entrevista na posição sentada, mantenha boa postura do tronco, evitando que este fique fletido, bem como não o mantenha em hiperextensão. Evite gestos e movimentos faciais, principalmente com sobrancelhas, boca e cabeça. 
2.3. Tenha cuidado com a aparência visual, no que diz respeito às suas roupas (devem estar limpas, passadas) e a aparência física (cabelos arrumados e barba feita, no caso dos homens), pois isso se traduz em maior confiabilidade para a população que irá assistir. Em relação ao vestuário é importante que obedeça certa formalidade, evitando roupas mais sensuais e muito coloridas.

2.4. Tenha calma durante sua explanação e tente ser o mais claro possível, com atenção para a correta expressão da língua portuguesa, sem utilização de siglas ou gírias e nunca de palavras obscenas ou "palavrões".

2.5. Utilize um tom de voz claro, nem muito alto, nem muito baixo, pois pode denotar arrogância ou timidez. Tenha em mente, que não existe "dono da verdade" e que sua missão é transmitir, de forma objetiva e clara, a informação para aquele momento, a qual auxiliará a sociedade de alguma forma.

2.6. Se cometer algum erro, peça para fazer uma nova entrevista, caso não seja ao vivo. Nos casos de entrevistas ao vivo, peça desculpas e corrija o erro. Tome cuidado com as perguntar sobre exercícios respiratórios e prevenção, ou algo que possa estigmatizar a profissão ou que não possua evidências científicas.

2.7. Ao final da entrevista agradeça a oportunidade e se coloque à disposição para qualquer esclarecimento.

\section{Pós-entrevista}

3.1. Em situações em que a entrevista não for gerada com a análise adequada, faça uma revisão da forma de resposta com o jornalista, para que a resposta seja transmitida de forma adequada.

3.2. Sempre que possível, solicitar cópia da entrevista e ou gravação para possível análise do que será publicado ou divulgado nos diferentes meios (TV, rádio, internet, jornal, ...).

3.3. Caso o que for veiculado tenha conteúdo distorcido ou diferente da realidade, envie requerimento oficial ao meio de comunicação utilizado, solicitando retratação e/ou correção.

\section{Considerações Finais}

Considerando a importância da assistência fisioterapêutica em pacientes com COVID-19 e a elevada demanda para realização de entrevistas pela imprensa, as presentes orientações visam subsidiar e alertar os fisioterapeutas respiratórios, cardiovasculares e intensivistas para os cuidados necessários nos momentos pré, durante e após a entrevista.

Outro ponto importante a se ressaltar, é que o fisioterapeuta deve ter atenção às normas do código de ética profissional, para que não ocorram infrações e as consequentes sanções decorrentes de processos éticos disciplinares, conforme prevê o Art. 17, da Lei no 6.316/1975 .

A divulgação de qualquer intervenção fisioterapêutica deverá ser respaldada por referências científicas, para que a Fisioterapia não seja banalizada com a divulgação de intervenções, as quais, não atinjam os efeitos desejados. 


\section{Referências}

1. Brasil. Resolução COFFITO nº 400, de 03 de agosto de 2011. Disciplina a Especialidade Profissional de Fisioterapia Respiratória e dá outras providências [Internet]. Brasília: Conselho Federal de Fisioterapia e Terapia Ocupacional; [2020]. Available from: https://www.coffito.gov.br/nsite/?p=3163

2. Brasil. Resolução COFFITO n 402 , de 03 de agosto de 2011. Disciplina a Especialidade Profissional Fisioterapia em Terapia Intensiva e dá outras providências [Internet]. Brasília: Conselho Federal de Fisioterapia e Terapia Ocupacional; [2020]. Available from: https://www.coffito.gov.br/nsite/?p=3165

3. Brasil. Resolução COFFITO no 454, de 25 de abril de 2015. Reconhece e disciplina a Especialidade Profissional de Fisioterapia Cardiovascular [Internet]. Brasília: Conselho Federal de Fisioterapia e Terapia Ocupacional; [2020]. Available from: https://www.coffito.gov.br/nsite/?p=3215

4. Thomas P, Baldwin C, Bissett B, Boden I, Gosselink R, Granger CL, et al. Physiotherapy management for COVID-19 in the acute hospital setting: clinical practice recommendations. J Physiother. 2020 Apr;66(2):73-82. doi: 10.1016/j.jphys.2020.03.011.

5. ANVISA. Resolução RDC No 07, de 24 de fevereiro de 2010. Dispõe sobre os requisitos mínimos para funcionamento de Unidades de Terapia Intensiva e dá outras providências. Brasília, DF: Agência Nacional de Vigilância Sanitária; [2020]. Available from: https://www20.anvisa.gov.br/segurancadopaciente/ index.php/legislacao/item/rdc-07-2010.

6. Kleinitz P, Mills JA, Connoly B, Skeiton P, Smith G, Clift Z. Considerações sobre a reabilitação durante o surto de COVID-19. Washington, DC: Organização Pan-Americana de Saúde (OPAS), Organização Mundial da Saúde (OMS); 2020. Available from: https://iris.paho.org/handle/10665.2/52103

7. Hospital Universitário da Universidade Federal do Maranhão. Guia de relacionamento com a imprensa [Internet]. São Luís: Assessoria de Comunicação do HUUFMA; [2020]. Available from: http://www2.ebserh.gov.br/documents/16424/2420545/GUIA+DE+RELACIONAMENTO+COM+A +IMPRENSA+20X20CM+-+ok.pdf/fef4c518-71cb-4ce8-be9d-e061763ad2d0.

8. Brasil. Resolução COFFITO n 424, de 08 de julho de 2002. Estabelece o Código de Ética e Deontologia da Fisioterapia [Internet]. Brasília: Conselho Federal de Fisioterapia e Terapia Ocupacional; [2020]. Available from: https://www.coffito.gov.br/nsite/?p=3187

9. Brasil. Lei no 6.316, de 17 de dezembro de 1975. Cria o Conselho Federal e os Conselhos Regionais de Fisioterapia e Terapia Ocupacional e dá outras providências [Internet]. Brasília, DF; 1975. Available from: http://www.planalto.gov.br/ccivil 03/leis/1970-1979/L6316.htm

Submissão em: 08/06/2020

Aceite em: 15/07/2020 